\title{
ON THE TRANSFORMATION GROUP OF A REAL HYPERSURFACE \\ BY
}

S. M. WEBSTER

\begin{abstract}
The group of biholomorphic transformations leaving fixed a strongly pseudoconvex real hypersurface in a complex manifold is a Lie group. In this paper it is shown that the Chern-Moser invariants must vanish if this group is noncompact and the hypersurface is compact. Also considered are transformation groups of flat hypersurfaces and intransitive groups.
\end{abstract}

Introduction. The purpose of this paper is to study the group of structure preserving, or pseudoconformal, transformations of a real hypersurface $M$ (of dimension $2 n+1$ ) in a complex $n+1$ manifold, or more generally, of a manifold with the same $C-R$ structure. We will always assume that the $C-R$ structure is integrable and that the Levi form is nondegenerate. It follows from the work of Chern and Moser [2] that this group is a Lie transformation group.

There is some similarity between the geometry of $M$ and the conformal geometry of a Riemannian manifold. Over $M$ there is a principal fibre bundle $B$ (the pseudoconformal bundle), a connection on $B$, and curvature invariants the most important of which is the fourth order curvature tensor $S_{\beta \rho \bar{\sigma}}^{\alpha}$ (see [2]). These have as analogues in the Riemannian case the bundle of conformal frames the conformal connection, and the Weyl conformal curvature tensor. If $n \geqslant 2, S$ vanishes if and only if $M$ is pseudoconformally flat, i.e., locally equivalent to the standard sphere $S^{2 n+1}$ in $\mathbf{C}^{n+1}$ [8].

Locally the structure of $M$ is given by a real one-form $\theta, n$ complex one-forms $\theta^{\alpha}$, and their complex conjugates $\theta^{\bar{\alpha}}=\overline{\theta^{\alpha}}$. They satisfy

$$
\theta \wedge \theta^{1} \wedge \cdots \wedge \theta^{n} \wedge \theta^{\overline{1}} \wedge \cdots \wedge \theta^{\bar{n}} \neq 0
$$

and the equation

$$
d \theta \equiv i g_{\alpha \bar{\beta}} \theta^{\alpha} \wedge \theta^{\bar{\beta}} \quad \bmod \theta .
$$

Here and throughout this paper Greek indices run from 1 to $n$ and the summation convention is used. The nondegenerate hermitian matrix $\left(g_{\alpha \bar{\beta}}\right)$ is

Received by the editors February 12, 1976.

AMS (MOS) subject classifications (1970). Primary 57E20, 53A55; Secondary 32F99.

Key words and phrases. Pseudoconformal transformation, Lie transformation group, strongly pseudoconvex, real hypersurface.

- American Mathematical Society 1977 
the Levi form. If $M$ is strongly pseudoconvex $\theta$ is chosen so that this matrix is negative definite. The holomorphic tangent bundle $H(M)$ is the annihilator of $\theta$ in the real tangent bundle of $M$. We will have need of the dual vector fields $X_{\alpha}, X_{\bar{\alpha}}=\bar{X}_{\alpha}$, and $X=\bar{X}$.

The real one-form $\theta$ is determined up to multiplication by a nonzero function on $M$. For a choice of $\theta$ the pair $(M, \theta)$, called a pseudohermitian manifold in [8], is the analogue of a Riemannian structure underlying a conformal structure.

In $\$ 1$ we show that if $M$ is compact, connected, and strongly pseudoconvex, and if the identity component of its pseudoconformal transformation group is noncompact, then $M$ is flat. The proof owes much to that given by Obata [7] in the conformal case.

In $\$ 2$ we study the unbounded one-parameter groups of pseudoconformal transformations of $S^{2 n+1}$. The main result is the same as in the conformal case [6]. This is used to show that, if $M$ is as in the preceding paragraph and has a finite fundamental group, then $M$ is globally equivalent to $S^{2 n+1}$. In $\$ 3$ we consider one-parameter groups with fixed points on a flat space.

We turn to intransitive groups of pseudohermitian transformations in $\S 4$. The theorem here is that if $G$ is an intransitive group of transformations of $(M, \theta), M$ having dimension $2 n+1$, then $G$ has dimension less than or equal to $n^{2}+1$. For $n=1$, Cartan [1] has shown that a three dimensional group of pseudoconformal transformations of a real hypersurface in $\mathbf{C}^{2}$ must be transitive.

The work presented in this paper was submitted as part of the author's thesis at the University of California at Berkeley in June of 1975.

1. Spaces admitting a noncompact connected Lie group of pseudoconformal transformations. In this section we prove the following:

THEOREM (1.1). Let $M$ be a compact, connected, strongly pseudoconvex abstract real hypersurface, and let $G$ be a connected Lie group of pseudoconformal transformations of $M$. If $G$ is noncompact then $M$ is locally equivalent to the standard real hypersphere $S^{2 n+1}$ in $C^{n+1}$ in the pseudoconformal sense.

This will be proved as follows. Suppose $M$ is not locally $S^{2 n+1}$; then we will show that the closure of every one-parameter subgroup of $G$ is compact. By a theorem of Montgomery and Zippin [5] $G$ must itself be compact.

We first assume that $\operatorname{dim} M=2 n+1, n \geqslant 2$.

Let $G_{1}$ be a one-parameter subgroup of $G$ with infinitesimal generator $Y$ on $M$. Assuming $M$ is not flat, it follows that the pseudoconformal curvature tensor $S_{\beta \rho \overline{\alpha \sigma}}$ is nonzero somewhere. Let $U$ be a nonempty connected component of the open set where $S$ does not vanish. $U$ is invariant under $G$, since $G$ is connected. 
Since $M$ is strongly pseudoconvex, we can choose a globally defined nonvanishing one-form $\theta$ annihilating the holomorphic tangent bundle $H(M)$. As in [8, §3], the one-form

$$
\theta^{*}=\|S\|_{\theta} \cdot \theta
$$

is a pseudoconformal invariant on $U$. Let $X^{*}$ be the corresponding invariant transversal [8]. The following Lie derivatives then vanish

$$
L_{Y} \theta^{*}=0, \quad L_{Y} X^{*}=\left[Y, X^{*}\right]=0 .
$$

On $U$ we can decompose $Y$ uniquely as

$$
Y=\eta X^{*}+\tilde{Y}
$$

where

$$
\eta=\theta^{*}(Y), \quad \theta^{*}(\tilde{Y})=0 .
$$

By the following proposition we may assume that $\eta$ does not vanish identically.

PROPOSITION (1.2). Let $Y$ be an infinitesimal pseudoconformal transformation on a nondegenerate, integrable $C-R$ manifold $M$. If $Y_{p}$ belongs to $H_{p}(M)$ for all $p$ in an open subset $U$ of $M$, then $Y$ vanishes identically.

Proof. Choose an local frame $\left(X, X_{\alpha}, X_{\bar{\alpha}}\right)$ and dual coframe $\left(\theta, \theta^{\alpha}, \theta^{\bar{\alpha}}\right)$. We have $d \theta=i g_{\alpha \bar{\beta}} \theta^{\alpha} \wedge \theta^{\bar{\beta}}+\theta \wedge \phi$, and we can put $Y=\xi^{\alpha} X_{\alpha}+\xi^{\bar{\alpha}} X_{\bar{\alpha}}$ in the open set $U$. Since $Y$ preserves the structure, we have

$$
L_{Y} \theta=u \theta \text {. }
$$

We also have

$$
L_{Y} \theta=\iota_{Y} d \theta+d \iota_{Y} \theta=i g_{\alpha \bar{\beta}} \xi^{\alpha} \theta^{\bar{\beta}}-i g_{\alpha \bar{\beta}} \xi^{\bar{\beta}} \theta^{\alpha}-\phi(Y) \theta .
$$

Comparing (1.5) and (1.6) we see that, for all $\beta, g_{\alpha \bar{\beta}} \xi^{\alpha}=0$. Since $g_{\alpha \bar{\beta}}$ is nondegenerate, $\xi^{\alpha}=0$ for all $\alpha$, and so $Y$ vanishes on $U$. The flow $G_{1}$ of $Y$ induces a flow $G_{1}^{\prime}$ on the pseudoconformal bundle $B$ over $M$ which preserves the connection forms. $G_{1}^{\prime}$ acts trivially on the part of $B$ over $U$, so by Theorem (3.2) of [3], $G_{1}^{\prime}$ consists of the identity alone. Therefore, $G_{1}$ is also the identity, and $Y$ vanishes everywhere on $M$.

Returning to the proof of Theorem (1.1), we may assume that $\eta>0$ somewhere by replacing $Y$ by $-Y$, if necessary. For a sufficiently small constant $\varepsilon>0$ we define a nonempty closed subset of $U$ by

$$
U_{\varepsilon}=\{p \in U: \eta(p) \geqslant \varepsilon\} .
$$

By (1.1) and (1.4) and the definition of $U$ it is seen that $\eta(p)$ goes to zero as $p$ approaches the boundary of $U$. Therefore, $U_{\varepsilon}$ is a closed subset of $M$ and so is compact.

$U_{\varepsilon}$ is invariant under $G_{1}$, the flow of $Y$, because 


$$
Y \eta=\left(L_{Y} \theta^{*}\right)(Y)+\theta^{*}([Y, Y])=0 .
$$

It is also invariant under the closure $\overline{G_{1}}$.

Let $P$ be the pseudohermitian bundle over $\left(U, \theta^{*}\right)$ with fibre $U(n)[8, \S 1]$. $P_{e}$, the part of $P$ lying over $U_{e}$, is compact and invariant by $\bar{G}_{1}$. By Theorem (3.2) of [3] and the fact that $\bar{G}_{1}$ is a Lie group, $\bar{G}_{1}$ imbeds as a closed submanifold of $P_{e}$. Therefore, $\bar{G}_{1}$ is compact, and Theorem (1.1) is true for $n>2$.

For the case $n=1$, we replace $\|S\|_{\theta}$ by the relative invariant $Q$ on $M^{3}$, the vanishing of which is necessary and sufficient for $M^{3}$ to be flat [1]. The same argument shows that Theorem (1.1) holds in this case also.

2. Unbounded one-parameter groups on the real hypersphere. To study further compact, strongly pseudoconvex spaces $M$ admitting a noncompact, connected Lie group of pseudoconformal transformations, we can restrict ourselves to the pseudoconformally flat spaces. It follows from the structure equations of $[2, \S 5]$ that $M$ can be developed locally onto the real hypersphere $S^{2 n+1}$. If $M$ is also simply connected, then the same monodromy argument as in the conformal case [4] shows that $M$ can be developed globally onto an open subset of $S^{2 n+1}$. This development may not be one-to-one.

A pseudoconformal vector field on $M$ will under this development be mapped to a pseudoconformal vector field on an open subset of $S^{2 n+1}$. Because $S^{2 n+1}$ has such a large group of transformations, any such local vector field extends to a unique global vector field. We therefore study one-parameter groups, particularly those with noncompact closure, on $S^{2 n+1}$. This is a matter of linear algebra.

In this section we will prove the following:

THEOREM (2.1). Let $G_{1}$ be a one-parameter group of pseudoconformal transformations of $S^{2 n+1}$ having a noncompact closure. Let $F_{t}$ be its flow. Then either

(1) $F_{t}$ has precisely two fixed points, $p_{-}$and $p_{+}$, such that for any other point $p$ in $S^{2 n+1}$,

$$
\lim _{t \rightarrow+\infty} F_{t}(p)=p_{+}, \quad \lim _{t \rightarrow-\infty} F_{t}(p)=p_{-} ;
$$

or

(2) $F_{t}$ has precisely one fixed point $p^{\prime}$, such that for any point $p$ in $S^{2 n+1}$,

$$
\lim _{t \rightarrow \pm \infty} F_{t}(p)=p^{\prime}
$$

Before giving the proof we will derive the following corollary.

Corollary (2.2). Let $M$ be a compact, connected, strongly pseudoconvex $C-R$ manifold with finite fundamental group. If $M$ admits a noncompact, connected Lie group $G$ of pseudoconformal transformations, then $M$ is globally equivalent to $S^{2 n+1}$. 
Proof. By Theorem (1.1), $M$ and hence its universal covering space $\tilde{M}_{\tilde{\sim}}$ are flat. Since $\tilde{M}$ is compact, the development map is a covering. Thus, $\tilde{M}$ is globally equivalent to $S^{2 n+1}$. Since $G$ is noncompact, it has a closed, noncompact one-parameter subgroup $G_{1}$; see [5]. $G_{1}$ lifts to a closed noncompact one-parameter subgroup $\tilde{G}_{1}$ on $\tilde{M}$. By Theorem (2.1), $\tilde{G}_{1}$ has either one or two fixed points, which must cover the fixed points of $G_{1}$. Therefore, $\tilde{M}$ is a one or two sheeted covering. If it were a two sheeted covering, both fixed points of $\tilde{G}_{1}$ would lie over the same fixed point of $G_{1}$. However, from case (1) of Theorem (2.1) these two fixed points have different character as $t \rightarrow+\infty$, one attracting and one repelling; hence, they could not cover the same fixed point of $G_{1}$. Therefore, $M \simeq \tilde{M} \simeq S^{2 n+1}$.

We go on to prove Theorem (2.1). As in [2] we view $S^{2 n+1}$ as a real hyperquadric in complex projective space. A pseudoconformal transformation of $S^{2 n+1}$ is induced by a projective transformation carrying $S^{2 n+1}$ into itself. Such a transformation is represented by a linear isomorphism $A$ of $C^{n+2}$, the space of homogeneous coordinates, which leaves invariant $\tilde{S}$, those coordinates of points in $S^{2 n+1}$. We denote by [Z] the point in projective space with homogeneous coordinates $Z$.

$S$ is defined by

$$
\tilde{S}=\left\{Z \in C^{n+2}:(Z, \bar{Z})=0\right\},
$$

where $\left(,^{-}\right)$is a nondegenerate hermitian form of signature $(n+1,1)$. We can view the pseudoconformal group of $S^{2 n+1}$ as $S U(n+1,1)$.

A one-parameter subgroup $A(t)$ of $S U(n+1,1)$ is given by

$$
A(t)=e^{t B} \text {. }
$$

where

$$
(B Z, \bar{W})+(Z, \overline{B W})=0, \quad Z, W \in C^{n+2} .
$$

Eigenvectors $Z$ of $B$ on $S$ correspond to fixed points [Z] of the group $A(t)$ acting on $S^{2 n+1}$.

Condition (2.3) readily implies the following:

Lemma (2.3). Let $Z$ be an eigenvector of $B$ with eigenvalue $\lambda$. If $Z$ is not in $\tilde{S}$, then $\lambda$ is purely imaginary.

LEMMA (2.4). Let $\left(^{-}\right.$, ) be an hermitian form on $C^{N+1}$, and $B$ be any operator satisfying (2.3). If $B$ has no eigenvectors on $\tilde{S}$, then $C^{N+1}$ has $a$ basis of eigenvectors with purely imaginary eigenvalues.

Proof. $B$ has some eigenvector, $B Z_{0}=\lambda_{0} Z_{0}$, and $\lambda_{0}+\bar{\lambda}_{0}=0$ by Lemma (2.3). By assumption $Z_{0} \notin\left(Z_{0}\right)^{\perp}$; hence, $C^{N+1}=\left(Z_{0}\right) \oplus\left(Z_{0}\right)^{\perp} .\left(Z_{0}\right)^{\perp}$ is invariant under $B$ by (2.3). We restrict $B$ and the hermitian form to $\left(Z_{0}\right)^{\perp}$, 
where, by induction, we have a basis $Z_{1}, \ldots, Z_{N}$ of eigenvectors with purely imaginary eigenvalues. $Z_{0}, Z_{1}, \ldots, Z_{N}$ is the required basis of $C^{N+1}$.

For the operator $B$ of Lemma (2.4) it follows that the corresponding one-parameter group $A(t)$ is bounded. Therefore, we assume that $B$ has at least one eigenvector on $\tilde{S}$. There are two cases.

Case (1). $B$ has at least two distinct eigenvectors $Z_{0}$ and $Z_{n+1}$ on $S$.

We must have $\left(Z_{0}, \bar{Z}_{n+1}\right) \neq 0$. Otherwise, $S$ would contain the complex 2-plane of all $a Z_{0}+b Z_{n+1}, a, b \in C$, and $S^{2 n+i}$ would contain a complex line, which it does not. We may assume that $\left(Z_{0}, \bar{Z}_{n+1}\right)=-i / 2$. From $(2.3)$, $Z_{0}$ and $Z_{n+1}$ have eigenvalues $\lambda$ and $-\bar{\lambda}$, respectively.

Choose $Z_{1}, \ldots, Z_{n}$, a basis of $\left(Z_{0}, Z_{n+1}\right)^{\perp}$. If relative to the basis $\left(Z_{0}, Z_{\alpha}\right.$, $\left.Z_{n+1}\right)$ of $C^{n+2}$ we have

$$
Z=\zeta^{a} Z_{a}, \quad 0 \leqslant a \leqslant n+1,
$$

then

$$
(Z, \bar{Z})=\zeta h^{t} \bar{\zeta}=h_{\alpha \bar{\beta}} \zeta^{\alpha \zeta \bar{\beta}}+\frac{i}{2}\left(\zeta^{n+1} \zeta^{\overline{0}}-\zeta^{0} \zeta^{\overline{n+1}}\right),
$$

where $h_{\alpha \bar{\beta}}$ is positive definite hermitian. (2.3) is now written

$$
B h+h^{t} \bar{B}=0 .
$$

From (2.6) it is seen that $B$ has the matrix form

$$
\left[\begin{array}{ccc}
\lambda & b^{\beta} & p \\
c_{\alpha} & B_{\alpha}^{\beta} & 2 i b_{\alpha} \\
q & \frac{1}{2 i} c^{\beta} & -\bar{\lambda}
\end{array}\right],
$$

where

$$
\begin{aligned}
& p=\bar{p}, \quad q=\bar{q}, \quad B_{\alpha}^{\gamma} h_{\gamma \bar{\beta}}+h_{\alpha \bar{\gamma}} B \overline{\bar{\gamma}}=0, \\
& c_{\alpha}=h_{\alpha \bar{\gamma}} \bar{\gamma}^{\bar{\gamma}}, \quad b_{\alpha}=h_{\alpha \bar{\gamma}} b^{\bar{\gamma}}, \quad \operatorname{trace} B=B_{\alpha}^{\alpha}+\lambda-\bar{\lambda}=0 .
\end{aligned}
$$

Since $Z_{0}$ and $Z_{n+1}$ are eigenvectors, we have

$$
p=q=c_{\alpha}=c^{\beta}=b_{\alpha}=b^{\beta}=0 .
$$

Since $h_{\alpha \bar{\beta}}$ is positive definite hermitian and the second relation of (2.8) holds, we may choose $Z_{1}, \ldots, Z_{n}$ so that both $\left(Z_{\alpha}, \bar{Z}_{\beta}\right)=\delta_{\alpha \bar{\beta}}$ and $B Z_{\alpha}=$ $\lambda_{\alpha} Z_{\alpha}$ where $\lambda_{\alpha}+\bar{\lambda}_{\alpha}=0$. As $A(t)$ is unbounded, we must have $\lambda=\mu+i \nu$, $\mu \neq 0$.

Let $Z \neq 0$ given by (2.4) represent a point [ $Z]$ in $S^{2 n+1}$ other than $\left[Z_{0}\right]$ and $\left[Z_{n+1}\right]$. From (2.5) it is seen that $\zeta^{0} \neq 0$ and $\zeta^{n+1} \neq 0$. The action of $A(t)$ is given by

$$
\left(\zeta^{0}, \zeta^{\alpha}, \zeta^{n+1}\right) \rightarrow\left(e^{t(\mu+i \nu)} \zeta^{0}, e^{i \lambda_{\zeta^{\alpha}}}, e^{-t(\mu-i v)} \zeta^{n+1}\right) .
$$


Let us assume $\mu>0$, so that as $t \rightarrow+\infty$ we have

$$
\left|e^{t(\mu+i v)} \zeta^{0}\right| \rightarrow+\infty, \quad\left|e^{-t(\mu-i v)} \zeta^{n+1}\right| \rightarrow 0,
$$

and the other components remain bounded. Therefore, as $t \rightarrow+\infty,[Z] \rightarrow$ $\left[Z_{0}\right]$. Also, as $t \rightarrow-\infty,[Z] \rightarrow\left[Z_{n+1}\right]$.

Case (2). $B$ has only one eigenvector $Z_{0}$ in $\tilde{S}$ with eigenvalue $\lambda_{0}$.

LEMma (2.5). If $B$ satisfies (2.6) and has $\lambda$ as an eigenvalue, it also has $-\bar{\lambda}$ as an eigenvalue.

Proof.

$$
\begin{aligned}
0 & =\operatorname{det}(B-\lambda I) \operatorname{det}(h)=\operatorname{det}(B h-\lambda h)=\operatorname{det}\left(-h^{t} \bar{B}-\lambda h\right) \\
& =\operatorname{det}(-h) \operatorname{det}^{t} \overline{(B+\bar{\lambda} I)} \cdot \square
\end{aligned}
$$

Let $W$ be an eigenvector with eigenvalue $-\bar{\lambda}_{0}$. We are assuming ( $W$, $\bar{W}) \neq 0$, so that $\lambda_{0}$ is purely imaginary, as are all the other eigenvalues. Let $\lambda_{0}, \lambda_{1}, \ldots, \lambda_{r}$ be the distinct eigenvalues of $B$ with eigenspaces $K_{0}$, $K_{1}, \ldots, K_{r}$, respectively.

LEMMA (2.6). $K_{0}+K_{1}+\cdots+K_{r} \subseteq\left(Z_{0}\right)^{\perp}$.

Proof. It follows from (2.3) that if $B Z_{j}=\lambda_{j} Z_{j}$ for $j>0$, then $\left(Z_{j}, \bar{Z}_{0}\right)=0$. Suppose $Z^{\prime} \in K_{0}$ and $\left(Z^{\prime}, \bar{Z}\right) \neq 0$, say $\left(Z^{\prime}, \bar{Z}_{0}\right)=1$. Put $Z=a Z_{0}+Z^{\prime}$, $a=\bar{a}$. Then $(Z, \bar{Z})=2 a+\left(Z^{\prime}, \bar{Z}^{\prime}\right)$, and we can choose $a$ so that $Z$ lies in $\tilde{S}$, contradicting the fact that $Z_{0}$ is the only eigenvector in $S$. Hence $K_{0} \subseteq\left(Z_{0}\right)^{\perp}$.

LEMMA (2.7). For any $Z$ in $C^{n+2}$ the complex line determined by the origin and $A(t) Z$ approaches the subspace $K_{0}+\cdots+K_{r}$ as $t \rightarrow \pm \infty$.

This will finish the proof of Theorem (2.1) as follows: since $S$ is invariant by $A(t)$ and $S \cap\left(K_{0}+\cdots+K_{r}\right)=\left(Z_{0}\right)$, we have, for any [Z] in $S^{2 n+1}$, $[A(t) Z] \rightarrow\left[Z_{0}\right]$ as $t \rightarrow \pm \infty$.

Proof. This will follow from the Jordan canonical form for $B$, according to which $C^{n+2}=V^{0} \oplus \cdots \oplus V^{r}$. The dimension $n_{j}$ of $V^{j}$ is the multiplicity of $\lambda_{j}$ in the characteristic polynomial of $B$, and each $V^{j}$ is invariant under $B$ and under $A(t)$. $B$ induces on each $V^{j}$ an operator $B_{j}$ of the form

$$
B_{j}=N_{j}+D_{j}, \quad N_{j} D_{j}=D_{j} N_{j}, \quad D_{j}=\lambda_{j} I_{n_{j}},
$$

where $N_{j}$ is nilpotent. $A(t)$ decomposes as $A(t)=e^{t B_{0}} \oplus \cdots \oplus e^{t B_{j}}$, and

$$
e^{i B_{j}}=e^{i \lambda_{j}}\left(I+t N_{j}+\cdots+\frac{t^{k}}{k !}\left(N_{j}\right)^{k}\right), \quad\left|e^{i \lambda_{j}}\right|=1
$$

where $k<n_{j}$ is such that $\left(N_{j}\right)^{k} \neq 0$ and all higher powers of $N$ vanish. 
Since any $Z$ in $C^{n+2}$ has the decomposition $Z=Z^{0}+\cdots+Z^{r}, Z^{j} \in$ $V^{j}$, it suffices to show that the complex line determined by the origin and $e^{t B_{j} Z^{j}}$ approaches $K_{j}$ as $t \rightarrow \pm \infty$. To simplify the notation assume $n_{j}=k+1$, so that $N_{j}$ has all ones below the main diagonal, and $V^{j}$ contains a unique (up to multiple) eigenvector $W^{j}=(1,0, \ldots, 0)$. Putting $Z^{j}=\left(\zeta^{0}, \ldots, \zeta^{k}\right)$, we have

$$
\begin{array}{r}
\zeta \cdot e^{t N}=\left(\zeta^{0}+\zeta^{1} t+\cdots+\zeta^{k} \frac{t^{k}}{k !}, \zeta^{1}+\cdots+\zeta^{k} \frac{t^{k-1}}{(k-1) !},\right. \\
\left.\ldots, \zeta^{k-1}+\zeta^{k} t, \zeta^{k}\right) .
\end{array}
$$

As $t \rightarrow \pm \infty$ the dominant term is in the first coordinate; hence, the line of $\zeta \cdot e^{t N}$ approaches the line of $W^{j}$.

Theorem (2.1) is proved.

3. One parameter groups on pseudoconformally flat spaces. In this section we will prove the following theorem.

THEOREM (3.1). Let $M$ be connected, strongly pseudoconvex, and pseudoconformally flat, and suppose $M$ admits a one-parameter Lie group $G_{1}$ of transformations which has a fixed point $p_{0}$. Then either $G_{1}$ is compact or $M$ is globally equivalent to $S^{2 n+1}$ or to $S^{2 n+1}$ with a point deleted.

Let $Y$ be the infinitesimal generator of $G_{1}$ on $M$. Develop a small neighborhood $U$ of $p_{0}$ onto an open subset $U^{\prime}$ of $S^{2 n+1}, p_{0}$ corresponding to $p_{0}^{\prime} .\left.Y\right|_{U}$ corresponds to a pseudoconformal vector field on $U^{\prime}$, which extends to a unique global $Y^{\prime}$ on $S^{2 n+1}$. There are three cases according to whether the one-parameter group $G_{1}^{\prime}$ of $Y^{\prime}$ is

(1) bounded, i.e., has compact closure $\bar{G}_{1}^{\prime}$ in $S U(n+1,1)$,

(2) unbounded with behavior (1) of Theorem (2.1), or

(3) unbounded with behavior (2) of Theorem (2.1).

Case (1). By integration over $\bar{G}_{1}^{\prime}, \bar{G}_{1}^{\prime}$ leaves invariant some $\theta^{\prime}$ and also the corresponding positive definite Riemannian metric

$$
d s^{2}=\theta^{\prime 2}-\operatorname{Re}\left(g_{\alpha \bar{\beta}}^{\prime} \theta^{\prime \alpha} \otimes \theta^{\prime \bar{\beta}}\right)
$$

on $S^{2 n+1}$. A small geodesic ball $B^{\prime}$ in $U^{\prime}$ centered at $p_{0}^{\prime}$ is mapped isometrically by each $g^{\prime}$ in $G_{1}^{\prime}$. Each $g$ in $G_{1}$ leaves the corresponding $\theta$ on $U$ invariant and maps the corresponding $B \subseteq U$ diffeomorphically.

Lemma (3.2). In Case (1), $G_{1}$ is compact.

Proof. We introduce the following notation: 
$\operatorname{PsC}\left(M, p_{0}\right)=$ the group of global pseudoconformal transformations of $M$ leaving $p_{0}$ fixed.

$\operatorname{PsC}\left(B, p_{0}\right)=$ the group of pseudoconformal transformations of $B$ leaving $p_{0}$ fixed.

$\operatorname{PsC}^{\prime}\left(B, p_{0}\right)=\operatorname{PsC}\left(B, p_{0}\right) \cap \operatorname{PsC}\left(M, p_{0}\right)$.

$\operatorname{PsH}\left(B, \theta, p_{0}\right)=$ the group of pseudohermitian transformations leaving $p_{0}$ fixed.

$\operatorname{PsH}\left(B, \theta, p_{0}\right)$ is compact. We have

$$
G_{1} \text { closed } \subseteq \operatorname{PsC}\left(M, p_{0}\right),
$$

since $G_{1}$ is a Lie subgroup; therefore,

$$
G_{1} \text { closed } \subseteq \operatorname{PsC}^{\prime}\left(B, p_{0}\right) .
$$

Since

$$
G_{1} \subseteq \operatorname{PsH}\left(B, \theta, p_{0}\right) \subseteq \operatorname{PsC}\left(B, p_{0}\right),
$$

it will suffice to show that $\operatorname{Ps}^{\prime}\left(B, p_{0}\right)$ is a closed subset of $\operatorname{Ps} C\left(B, p_{0}\right)$.

Let $\left\{\phi_{j}\right\}$ be a sequence in $\operatorname{PsC}^{\prime}\left(B, p_{0}\right)$ and suppose $\phi_{j}$ converges to $\phi$ in $\operatorname{PsC}\left(B, p_{0}\right)$. We must show that $\phi$ is globally defined on $M$. Choose a pseudoconformal frame $e_{0}$ at $p_{0}$. We have $\phi_{j} \cdot e_{0} \rightarrow \phi \cdot e_{0}$. By Theorem (3.2) of [3] the map $g \rightarrow g \cdot e_{0}$ embeds $\operatorname{PsC}(M)$ into the bundle $B$ of pseudoconformal frames as a closed submanifold. So there exists some $\phi_{0}$ in $\operatorname{PsC}(M)$ with $\phi_{0} \cdot e_{0}=\phi \cdot e_{0}$. Since $\phi$ and $\phi_{0}$ agree at $e_{0}$, we have $\phi_{\tilde{\nu}}=\phi_{0}$ on $B$.

Case (2). Pass to the universal covering $\tilde{M}$ of $M, \tilde{Y}$ covering $Y$ and $p_{1}$ lying over $p_{0}$. Let $f$ be development of $M$ over $S^{2 n+1}$. Let $p_{1}$ correspond to $p_{-}$of Theorem (2.1) and $\tilde{Y}$ correspond to $Y^{\prime}$ under $f$. Define

$$
V=\left\{p_{1}\right\} \cup\left\{\text { all orbits of } \tilde{Y} \text { leaving } p_{1}\right\} .
$$

Since $Y$ and hence $\tilde{Y}$ are complete vector fields, $f$ maps $V$ one-to-one onto $S^{2 n+1}-\left(p_{+}\right)$. Suppose $p_{2}$ is in $\operatorname{bdry}(V)$; then $f\left(p_{2}\right)=p_{+}$. Since $f$ is a local diffeomorphism, bdry $(V)$ is discrete, and so $V$ has no exterior. Furthermore, $\tilde{Y}$ has exactly one zero of type $p_{-}$. Interchanging $p_{2}$ and $p_{1}$ in this argument shows that $\tilde{Y}$ has exactly one zero of type $p_{+}$also. So, either bdry $(V)=\left\{p_{2}\right\}$ and $M \simeq S^{2 n+1}$, or bdry $(V)=\varnothing$ and $M \simeq S^{2 n+1}-\left\{p_{+}\right\}$. In the latter case $\tilde{Y}$ has only one zero, so $\tilde{M}$ is a one sheeted covering. In the former case $\tilde{Y}$ has two zeroes of opposite character, so again $\tilde{M}$ is a one sheeted covering. Case (3). We proceed as in Case (2) except that there is only one zero $p^{\prime}$ of $Y^{\prime}$ and $p^{\prime}=f\left(p_{1}\right)$. Let

$$
U=\left\{\text { all orbits of } Y \text { leaving } p_{1}\right\} .
$$

Again, since $\tilde{Y}$ is complete, $f$ maps $U$ one-to-one onto $S^{2 n+1}-\left\{p^{\prime}\right\}$, $\operatorname{bdry}(U)$ $\subseteq f^{-1}\left(p^{\prime}\right)$ is discrete, and $U$ has no exterior. For any $p_{2}$ in $U \lim _{t \rightarrow-\infty} \phi_{t}\left(p_{2}\right)$ $=p_{1}$, where $\phi_{t}$ is the flow of $\tilde{Y}$. Suppose also $f\left(p_{1}^{*}\right)=p^{\prime}$. Then for any $p_{2}^{*}$ in 
$U^{*}, \lim _{t \rightarrow-\infty} \phi_{t}\left(p_{2}^{*}\right)=p_{1}^{*}$. As $M$ is connected, $U \cap U^{*} \neq \varnothing$, so we have $p_{1}=p_{1}^{*}$. Therefore, $f$ maps $\tilde{M}=U \cup\left\{p_{1}\right\}$ one-to-one and onto $S^{2 n+1}$. Since $\tilde{Y}$ has only one zero, $M \simeq \tilde{M} \simeq S^{2 n+1}$.

This proves Theorem (3.1).

In view of Theorem (3.1) it follows that if $M$ satisfies the hypothesis of Theorem (1.1), then either $M \cong S^{2 n+1}$ or every closed noncompact oneparameter subgroup on $M$ has no fixed points. In this respect let us note the following:

Proposition (3.3). Suppose $G_{1}$ is a one-parameter Lie group of pseudoconformal transformations of $M$ as in Theorem (1.1), and let $Y$ be its infinitesimal generator. If $Y$ is never tangent to $H(M)$, then $G_{1}$ is compact.

Proof. Choose some $\theta$ defining $H(M)$. Let $\eta=\theta(Y)$. $\eta$ is never zero, since $Y$ is always transversal. We have $L_{Y} \theta=u \theta$, so that $L_{Y} \eta=u \eta$, and $L_{Y}\left(\eta^{-1} \theta\right)=0$. $G_{1}$ leaves $\theta^{*}=\eta^{-1} \theta$ invariant, so must be compact by Theorem (1.2) of [8].

4. Intransitive groups. In this section we prove the following theorem.

THEOREM (4.1). Let $G$ be an effective Lie group of pseudohermitian transformations of a strongly pseudoconvex, connected, $C$ - $R$ manifold $(M, \theta)$ of dimension $2 n+1$. If $G$ is not transitive then the dimension of $G$ is less than or equal to $n^{2}+1$.

EXAmple (4.2). To see that the estimate on $\operatorname{dim} G$ is the best possible, consider $S^{2 n+1} \subseteq C^{n+1}$ with $\theta=i \partial r$, where $r=z^{1} z^{\overline{1}}+\cdots+z^{n} z^{\bar{n}}+w \bar{w}-$ 1. The group is $G=U(n)+U(1)$ acting on $C^{n+1}$ by

$$
\left(z^{\alpha}, w\right) \rightarrow\left(z^{\beta} U_{\beta}^{\alpha}, w \mu\right), \quad U^{t} \bar{U}=I, \mu \bar{\mu}=1 .
$$

This group preserves $r$ and hence $\theta$ and is clearly intransitive.

To prove Theorem (4.1) we recall that relative to an admissible coframe as in [8], we have

$$
d \theta=i g_{\alpha \bar{\beta}} \theta^{\alpha} \wedge \theta^{\bar{\beta}}
$$

On $H(M)$ we have the negative definite hermitian form

$$
h=g_{\alpha \bar{\beta}} \theta^{\alpha} \theta^{\bar{\beta}} \text {. }
$$

This form is invariant under the action of $G$. For a point $p$ in $M$ we use the notation

$$
G(p)=\text { orbit of } p \text { under } G
$$

and

$$
G_{p}=\text { isotropy subgroup of } p,
$$


so that $G(p)=G / G_{p}$. We also define $W_{p}=T_{p}(G(p)) \cap H_{p}(M)$, and

$J=$ complex multiplication on $H_{p}(M)$.

$W_{p}$ is a real subspace of $H_{p}$, so $0 \leqslant \operatorname{dim} W_{p} \leqslant 2 n$, and is invariant under the action of $G_{p}$. We have a faithful representation of $G_{p}$ on $U(n)$, the unitary group of $\left(H_{p}, h, J\right)$.

The proof is divided into four parts.

(1) Suppose $W_{p}=0$ for some $p$ in $M$. Then $\operatorname{dim} G(p) \leqslant 1$ and $\operatorname{dim} G=$ $\operatorname{dim} G(p)+\operatorname{dim} G_{p} \leqslant 1+n^{2}$.

(2) Claim. For any $p$ in $M, \operatorname{dim} W_{p}<2 n$, i.e., $W_{p} \neq H_{p}$, If $W_{p}=H_{p}$, then two cases are possible:

(a) $T_{p}(G(p))=T_{p}(M)$. Then $G$ is locally transitive at $p$ and $G(p)$ is an open subset of $M$. Since the pseudohermitian bundle has compact fibres $U(n)$ and $G$ is a Lie transformation group, $G(p)$ is also closed in $M$. So $G(p)=M$, contradicting the fact that $G$ is not transitive.

(b) $T_{p}(G(p))=H_{p}(M)$. Then $G(p)$ is a maximal integral submanifold of $\theta=0$. Therefore $d \theta$ vanishes when restricted to $G(p)$, contradicting the fact that the form (4.2) is negative definite on $H_{p}(M)$.

So we may assume that $0<\operatorname{dim} W_{p}<2 n$ for all $p$ in $M$. Let us denote $V_{p}=W_{p} \cap J W_{p}$, and $K_{p}=W_{p}+J W_{p}$. These are $G_{p}$ invariant complex subspaces of $H_{p}(M)$.

(3) Suppose that, for some $p$ in $M, W_{p}$ is not a totally real subspace of $H_{p}$, i.e., $V_{p} \neq 0$. We get a nontrivial $G_{p}$ invariant decomposition $H_{p}=V_{p} \oplus V_{p}^{\perp}$. Letting $l=\operatorname{dim}_{C} V_{p}$, we have $G_{p} \subseteq U(l) \oplus U(n-l)$. So

$$
\operatorname{dim} G_{p} \leqslant l^{2}+(n-l)^{2} \leqslant 1+(n-1)^{2},
$$

and

$$
\operatorname{dim} G \leqslant 2 n+1+(n-1)^{2}=n^{2}+2 .
$$

If equality holds in the last estimate, then $\operatorname{dim} G(p)=2 n$ and $\operatorname{dim} G_{p}=1$ $+(n-1)^{2}$. The complex dimension of $V_{p}$ is then $n-1$, and $G_{p} \simeq U(n-1)$ $\oplus U(1)$. Since the dimension of $W_{p}$ is at most one less than $\operatorname{dim} G(p)$, we have $\operatorname{dim} W_{p}=2 n-1$. We have $G_{p}$ invariant decompositions $W_{p}=V_{p} \oplus R$, and $H_{p}=V_{p} \oplus(R+J R)$, where $R$ is a one dimensional subspace. It follows that $G_{p} \subseteq U(n-1) \oplus O(1, R)$, implying that $\operatorname{dim} G_{p} \leqslant(n-1)^{2}$, a contradiction. Hence $\operatorname{dim} G<n^{2}+1$.

(4) Suppose that, for some $p$ in $M, W_{p}$ is nontrivial and totally real. We then have $\operatorname{dim} W_{p}=k \leqslant n$, and $K_{p}$ is of complex dimension $k$. We have the $G_{p}$ invariant decompositions $H_{p}=K_{p} \oplus K_{p}^{\perp}$, and $K_{p}=W_{p} \oplus J W_{p}$. Therefore,

$$
\operatorname{dim} G_{p} \leqslant \frac{1}{2} k(k-1)+(n-k)^{2},
$$

and 


$$
\begin{aligned}
\operatorname{dim} G & \leqslant(k+1)+\frac{1}{2} k(k-1)+(n-k)^{2} \\
& \leqslant n^{2}+1-\frac{1}{2} k^{2}+\frac{1}{2} k \leqslant n^{2}+1,
\end{aligned}
$$

since $k<n$.

Theorem (4.1) is proved.

\section{REFERENCES}

1 a. E. Cartan, Sur la géométrie pseudo-conforme des hypersurfaces de l'espace de deux variables complexes. I, Ann. Mat. Pura Appl. (4) 11 (1932); 17-90.

b. Sur la géométrie pseudo-conforme des hypersurfaces de l'espace de deux variables complexes. II, Ann. Scuola Norm. Sup. Pisa (2) 1 (1932), 333-354.

2. S. S. Chern and J. Moser, Real hypersurfaces in complex manifolds, Acta Math. 133 (1974), 219-271.

3. S. Kobayashi, Transformation groups in differential geometry, Springer-Verlag, Berlin and New York, 1972. MR 50 \#8360.

4. N. H. Kuiper, On conformally-flat spaces in the large, Ann. of Math. (2) 50 (1949), 916-924. MR 11, 133.

5. D. Montgomery and L. Zippin, Existence of subgroups isomorphic to the real numbers, Ann. of Math. (2) 53 (1951), 298-326. MR 13, 911.

6. M. Obata, Conformal transformations of Riemannian manifolds, J. Differential Geometry 4 (1970), 331-333. MR 42 \#2387.

7. The conjectures on conformal transformations of Riemannian manifolds, J. Differential Geometry 6 (1971/72), 247-258. MR 46 \#2601.

8. S. Webster, Pseudo-Hermitian structures on a real hypersurface, J. Differential Geometry (to appear).

Department of Mathematics, Princeton University, Princeton, New Jersey 08540 